\title{
Implementación de un Programa de Módulos Didácticos que Contribuya para Desarrollar la Capacidad Lógico-Matemática en Estudiantes del Nivel Secundario de las Instituciones Educativas de Tacna
}

RESUMEN. En nuestra propuesta, los esfuerzos están encaminados en aportar a la educación de la región y del país, con la producción de módulos didácticos para capacitar y actualizar en: Lógico Matemática, y que permitan el desarrollo de competencias, estándares, actitudes y valores, con estrategias de aprendizaje descubriendo los elementos de cada subtema, elaborando definiciones, descubriendo el proceso de realización, para motivar a los estudiantes y llevar esta ciencia a la solución de los problemas de la vida diaria.

El presente trabajo implementa módulos educativos, planteando situaciones problemáticas y tratamiento empírico, para luego ser abordadas con un desarrollo teórico cientifico que servirá de algoritmo para la solución y aplicación en diversos problemas concretos de la vida diaria.

\begin{abstract}
In our proposal, the efforts are directed in contributing to the education of the region and the country, with the production of didactic modules to enable and to update in: Logical Mathematician, and that they allow to the development of competitions, standards, attitudes and values, with learning strategies discovering the elements of each subsubject, constructing definitions, discovering the accomplishment process, to motivate the students and to take this science to the solution of the problems of the daily life.
\end{abstract}

The present work implements educative modules, raising problematic situations and empirical treatment, soon to be boarded with a scientific theoretical development that will serve as algorithm for the solution and application of diverse concrete problems of the daily life.

\section{INTRODUCCIÓN. En los sucesivos módulos} didácticos presentados, vamos a plantearnos algunas cuestiones relativas al desarrollo del pensamiento matemático, de nuestro pensamiento matemático. Pero no se trata de un trabajo abstracto. Este material nace de las dificultades detectadas en los procesos de formación de nuestros estudiantes de Educación Básica Regular, y va dirigido a los maestros y maestras que vivimos con el ideal de elevar la calidad educativa. Es decir, a los que asumimos como misión educativa "formar a los niños, niñas, jóvenes y adultos de los sectores más necesitados de nuestra región.

Con nuestra propuesta pretendemos colaborar en la formación de un estudiante capaz de generar procesos de cambio y transformación social; reflexivo y con capacidad para potenciar el diálogo de saberes y el discernimiento creativo, indispensable para inventar y seguir inventando nuevas ideas y formas de alcanzar la realización de esa sociedad y de ese sujeto deseado.

\section{MATERIAL Y MÉTODOS}

El presente trabajo implementa módulos educativos, planteando situaciones problemáticas y tratamiento empírico, para luego ser abordadas con un desarrollo teórico científico que servirá de algoritmo para la solución y aplicación de diversos problemas concretos de la vida diaria.

\section{RESULTADOS}

Presentamos la implementación de uno de los módulos didácticos:

\section{MÓDULOVI: Fracciones decimales}

INTRODUCCIÓN. La matemática constituye uno de los grandes logros de la inteligencia humana conformando un aspecto medular de la cultura contemporánea, un poderoso sistema teórico de alto nivel de abstracción, es decir, potencialmente de muchísima utilidad.

El trabajo del maestro en una sesión de aprendizaje de matemática es de suma importancia, ya que debe servir de mediador entre el conocimiento matemático y el aprendizaje de sus estudiantes, proporcionándoles los instrumentos necesarios para potenciar su desarrollo en el área de la matemática, así como las actitudes positivas hacia el área.

1. En este sentido, el módulo ilustra de manera sencilla e instructiva todo lo referente a fracciones decimales.

Esperamos contribuir en esta importante área del conocimiento: la matemática. 


\section{ÍNDICE}

\section{INTRODUCCIÓN}

Situaciones problemáticas

Desarrollo del contenido Teórico Cientifico

¿Qué son las fracciones?

Números decimales

Números racionales

Actividades de Aplicación

Problemas propuestos

Bibliografia

\section{Situaciones Problemáticas}

A) ¿De cuántos kilates era la joya?, generalmente contestan con un tímido debía ser de 24 , no?

Pues bien, de 24 kilates seguramente no sería, porque si bien el oro es muy apropiado para la fabricación de joyería por ser el más maleable de todos los metales, en estado puro ( 24 kilates) es demasiado dúctil y blando, por lo que se hace necesaria su aleación con otros metales para darle el grado de dureza necesario para la elaboración de joyas.

Los kilates nos indican la proporción de oro y la de otros metales que contiene la joya, lo que influye de forma determinante en su precio. Así, para saber si estamos ante una ganga o no, lo primero que tenemos que hacer es conocer su "kilataje".

- 24 kilates $(1000$ milésimas $)=100,00 \%$ oro puro

- 18 kilates ( 750 milésimas $)=75,00 \%$ oro puro

- 14 kilates $(585$ milésimas $)=58,33 \%$ oro puro

- 10 kilates $(417$ milésimas $)=41,66 \%$ oro puro

Así pues lógicamente cuanta mayor sea su proporción en oro más elevado será el precio de la joya.

Ejemplo: una joya de 16 gramos de oro de 14 kilates posee la cantidad de $9,33 \mathrm{~g}$ de oro puro. Compruebe dicho resultado e indique cuántos gramos no son de oro...

B) Podemos observar algunos otros ejemplos sobre el uso de fracciones en la vida diaria:

Me serví medio litro de refresco.

Ojala no llueva, ya que para ir de Tacna a Puno necesitamos medio día.

Mi casa está en la mitad de la cuadra.

Puse el cuarto de kilo de queso en un envase.

Queda un poco menos que tres cuartos de

litro de aceite.

Ese señor pidió un quinto de veinte mangos.

Tranquilo, que solo son cuatro mangos.

C) En una receta de un libro de cocina se puede encontrar instrucciones siguientes:
$1 / 2$ kilo de maíz

1 pollo de 1 kilo y $1 / 2$

$1 / 2$ pimentón

Calentar el horno $1 / 4$ de hora previamente

Cocinar durante $3 / 4$ de hora

\section{Desarrollo del Contenido Teórico Científico}

¿Qué son las fracciones?

Se llaman así a todos los números que representen una cantidad inexacta, por lo general vienen de una división inexacta.

Por ejemplo:

$$
\begin{aligned}
& 8 \div 5=\frac{8}{5} ; \frac{4}{6} ; \frac{79}{36} \quad \text { Son fracciones ordinarias } \\
& \frac{117}{100} ; 1 / 100 ; 71 \% . \text { Son fracciones decimales }
\end{aligned}
$$

\section{Números Decimales}

Se llaman así a todos los números que representen una cantidad inexacta, y que presentan una parte entera y una parte decimal, las mismas que se encuentran separadas por la coma decimal (,). Por ejemplo:

$$
\begin{aligned}
& 0.65 \text { Este es un número decimal } \\
& \begin{array}{l}
0,65 \text { La parte entera se encuentra a la izquierça de la coma decimal, en este caso } \\
\text { será } 0 .
\end{array} \\
& 0.65 \text { La parte decimal se encuentra a la derecha de la coma decimal, en este caso } \\
& \text { será } 65 \text {. }
\end{aligned}
$$

Ejemplos:

1) $1 / 2=0,5$ que es el resultado de dividir $1: 2$

2) $1 / 3=0,333 \ldots$ que es el resultado de dividir $1: 3$

3) $1 / 4=0,25$ que es el resultado de dividir 1:4

4) $7 / 15=, 4666$ que es el resultado de dividir $7: 5$

\section{Situaciones Problemáticas}

1. En una clase del CEID hay 30 participantes, de los cuales los $3 / 5$ son damas. ¿Cuántas damas hay en esta clase? ¿Y cuántos varones?

2. Kelvin ayuda a su papá en su negocio. Durante las vacaciones lo hace de lunes a viernes y en época de clases, los sábados. Por cada día de trabajo recibe $\mathrm{S} / .4,50$. Al terminar las 8 semanas de vacaciones había ganado $2 / 3$ del dinero que necesita para comprarse una bicicleta nueva. ¿En cuántos 
sábados reunirá lo que le falta? ¿Cuánto cuesta la bicicleta que quiere comprar?

3. Perla gastó en el mercado mayorista las tres cuartas partes del dinero que llevaba. Después se dirigió a comprar tres pares de zapatillas a S/. 70 cada una, pero le faltaban S/. 20. ¿Cuánto dinero tenía al entrar al mercado mayorista?

\section{Números Racionales}

Los racionales son números que pueden expresarse como fracción:

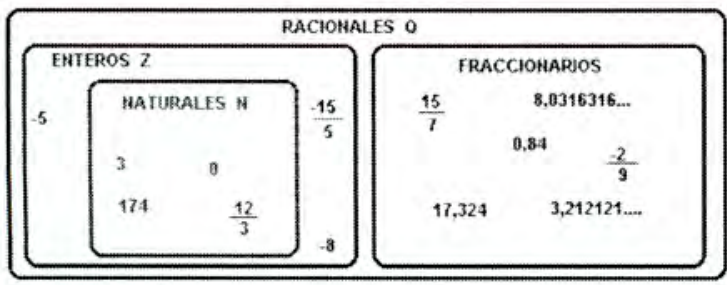

Figura 1: Conjunto de los números racionales.

También pertenecen a los números racionales los números enteros: $8,-5,56,0$, cuyo denominador es el 1 , el cual no se escribe. Por lo tanto, el conjunto Q de los racionales tiene subconjunto a los enteros $(\mathrm{Z})$, y los Naturales (N)

Los Irracionales en cambio son aquellos números que no pueden ser escritos en forma fraccionaria, por ejemplo: los números decimales infinitos no-periódicos, raíces no exactas y algunas constantes. Por ejemplo:

$\pi=3,1415 \ldots$,

$\mathrm{e}=2,717 \ldots, \sqrt{2}=1,4142 \ldots$

La unión de los racionales (Q) y los Irracionales (I) da como resultado un nuevo conjunto denominado: Números Reales (R).

Clasificación de los Racionales: Los números racionales pueden representarse como fracciones comunes o como decimal.

\section{Situaciones Problemáticas}

1. A primeros de diciembre, un ciclista pesaba $72,5 \mathrm{~kg}$. $\mathrm{y}$ en ese mes engordó $1,375 \mathrm{~kg}$. ¿Cuánto pesaba a primeros de febrero si en enero adelgazó $2,26 \mathrm{~kg}$ ?

2. Marcos y Marcelo tienen que repartir S/.12 entre 10 amigos. Para saber cuánto le toca a cada uno, hacen el cálculo 12:10. Indique usted otros procedimientos.

3. Cuando Luis llegó de la I.E. su mamá le dijo que no prendiera la tele hasta las 19 horas. Luis llegó de la I.E. a las 17 horas. Tardó 0,25 de hora en tomar la leche y le dedicó 1 hora a hacer la tarea. Esperó media hora más y prendió la tele. ¿Te parece que obedeció a su mamá? ¿Por qué? Si consideras que no obedeció, ¿cuánto tiempo más tendría que haber esperado?
4. Un corredor debe realizar la carrera de 100 metros. En la pista hay marcas, todas a la misma distancia unas de otras. A continuación, una representación de la pista:

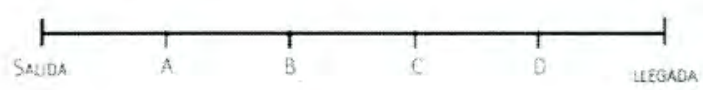

Contesta las preguntas y explica cómo pensaste cada respuesta.

a) Cuando el corredor está en el punto $\mathrm{B}$ ¿qué fracción del total del camino habrá recorrido? ¿Y cuántos metros recorrió?

b) Cuando el corredor haya recorrido tres quintos del trayecto, ¿dónde estará?

c) Cuando el corredor esté en el punto D, ¿qué fracción del total habrá recorrido?

d) ¿Cuántos metros habrá recorrido cuando se encuentre en el punto $\mathrm{A}$ ?

e) Si el corredor se encuentra a los $80 \mathrm{~m}$ de la salida, ¿en qué punto está?

\section{ACTIVIDADES DE APLICACIÓN}

\section{Primera parte: Fracciones decimales}

- Presentación de situaciones problemáticas de la vida diaria.

- Relacionar las situaciones planteadas con las fracciones decimales.

- Exploración de saberes previos.

\section{Segunda parte: Números Decimales}

- Resuelven las situaciones problemáticas planteadas en la hoja de práctica, en equipos de 4 integrantes y lo presentan en la pizarra.

- Contrastan las informaciones obtenidas con la información presentada en el modulo.

- Resuelven los ejercicios propuestos.

- Reconocen los números racionales.

\section{Tercera parte: producto final}

En equipos de 4 integrantes, proponen dos situaciones problemáticas relacionadas con su área de trabajo, resuelven y los sustentan, aplicando los contenidos de fracciones y números decimales.

Para realizar el producto tener en cuenta los indicadores de evaluación que presentamos a continuación. 


\begin{tabular}{|c|c|c|c|c|}
\hline \multicolumn{5}{|c|}{ INDIC ADORES DE EVALUACIÓN DEL PRODUCTO } \\
\hline \multirow[t]{2}{*}{ Indicadores } & \multirow[t]{2}{*}{ Puntaje } & \multicolumn{2}{|c|}{$\begin{array}{c}\text { valoraci } \\
\text { on }\end{array}$} & \multirow{2}{*}{$\begin{array}{l}\text { No } \\
\text { ta }\end{array}$} \\
\hline & & B & R $\mathbf{M}$ & \\
\hline $\begin{array}{l}\text { Formulación Correcta de las situaciones } \\
\text { problemáticas. }\end{array}$ & 4 & & & \\
\hline $\begin{array}{l}\text { Aplica adecuadamente las operaciones con numeros } \\
\text { fraccionarios. }\end{array}$ & 4 & & & \\
\hline $\begin{array}{l}\text { Aplica adecuadamente las operaciones con numeros } \\
\text { fraccionarios. }\end{array}$ & 4 & & & \\
\hline $\begin{array}{l}\text { Determinación correcta de la solución de las } \\
\text { situaciones problemáticas de la vida cotidiana. }\end{array}$ & 4 & & & \\
\hline Prescntación y organización del producto. & 4 & & & \\
\hline PUNTAJES PARCIALES & 20 & & & \\
\hline
\end{tabular}

\section{PROBLEMAS PROPUESTOS}

1. Ya completé los $2 / 5$ de mi álbum de fotografias. Para llenar un cuarto de lo que me falta necesito 36 fotografias. ¿Cuál es la capacidad de mi álbum?

2. Un grupo de profesores caminan $8 / 5 \mathrm{~h}$; luego descansan $2 / 3$ de hora; vuelven a caminar $1 / 4$ de hora, descansan $1 / 2$ hora y emprenden la última jornada de 3/2 de hora hasta llegar a su meta. ¿Qué tiempo caminaron?

3. He gastado los $4 / 7$ del dinero que tenía más $5 / 9$ del resto. ¿Cuánto tenía si aún me quedan 24 nuevos soles?

4. Para comprar un cuaderno de $\mathrm{S} / .58,60 \mathrm{me}$ falta $\mathrm{S} /$. 20,50; pero para comprar un libro me falta $S / .88,25$. ¿Cuánto cuesta el libro?

5. La docena de cuadernos cuesta S/. 42,50; si se vende 2 cuadernos por $\mathrm{S} / .8,30$, ¿cuántas docenas hay que vender para ganar $\mathrm{S} / .43,80$ ?

6. En una encuesta aplicada en una I.E. se determinó que los $3 / 5$ de los profesores tienen "Internet" en sus domicilios y los 3/10 tienen "TV. por cable". Se sabe que los que tienen Internet o TV. cable, pero no ambos constituyen el $7 / 10$ de la plana docente $y$ hay 400 profesores que no tienen acceso a ninguno de los servicios precitados. ¿Cuántos profesores tienen ambos servicios?

7. Una vendedora de huevos vendió la cuarta parte del número de huevos que tenía a $\mathrm{S} / .0,20$ cada uno y la novena parte a S/. 0,18 cada uno. Sí obtuvo por las dos ventas entre 8 y 12 nuevos soles, ¿cuántos huevos tenía inicialmente?

\section{CONCLUSIONES}

1. Los momentos que vivimos nos están consolidando en una sociedad del conocimiento; en ella la gestión de los distintos saberes es la que determina el accionar humano, la competitividad, las formas y niveles de producción, etc.

2. La creación y aplicación de los módulos didácticos han llegado a ser de tal magnitud que prácticamente se convierten en un instrumento esencial de los estudiantes de Educación Básica Regular.

3. Los nuevos módulos didácticos de lógico matemática estarán en relación con tareas inteligentes que exigen un nivel de adaptación y de creatividad. En definitiva, parece que más que disminuir, aumentará el papel del factor humano y cobrará especial importancia una formación adecuada que permita asumir los nuevos retos que se presentan como ineludibles.

4. Es fácil comprender que los módulos educativos se conviertan en sistemas estratégicos para los estudiantes de la Educación Básica Regular.

\section{REFERENCIA BIBLIOGRÁFICA}

FIGUEROA, R. (1986). Matemática Básica I. Lima.

COVEÑAS, M (2006). Matemática l. Educación Secundaria (3ra.edic.), Lima, Bruño.

RUIZ,I. (1998). Aritmética (1 ra.edic.) Lima, Coveñas.

SANTIBÁÑEZ, J. (1991). Aritmética. Colección Euclides, Lima.

GALDÓS, L. (2005). Dominando las Matemáticas: Aritmética I. ( ${ }^{\mathrm{a}}$ edic.), Lima, Quevecor World Perú S.A.

SILVA, M. (2000). Aritmética. (1 $1^{\text {a }}$ edic.). Lima, San Marcos.

HERNÁNDEZ, H. y otros (2005). Aritmética. ( $1^{\mathrm{a}}$ edic.). Lima, Proyecto Ingenio.

SALVADOR, Timoteo. Razonamiento matemático. Siglo XXI, Editorial San Marcos, Lima, 2003.

N COVEÑAS, M. Matemática 1 - Educación Secundaria. $3^{a}$ edición, Lima, Bruño, 2006 )

RUBIÑOS T., Luis, Razonamiento matemático. Editorial Moshera, Lima, 2003.

SANTIBÁÑEZ, José. Aritmética. Colección Euclides, Lima, 1991. 